\title{
Traffic Predicting Model for Dynamic Spectrum Sharing Over 5G Networks
}

\author{
Ahmed Alshaflut, Vijey Thayananthan \\ Faculty of Computing and IT \\ King Abdulaziz University, \\ Jeddah, Saudi Arabia
}

\begin{abstract}
Recently, wireless networks and traffic requirements have been rapidly aggregated in diverse applications in $5 \mathrm{G}$ environments. For this reason, researchers have investigated the influences of this growth based on a user's requirements inside these networks. However, the stream of traffic has been considered a crucial role for the user's needs over $5 \mathrm{G}$ network. In this paper, gigantic data traffic is considered for enabling dynamic spectrum sharing over 5G networks. Thus, various accessing plans are covered to manage the overall network traffic. Additionally, it proposes a traffic predicting model for a technique of managing traffic when multiple requests are received to decrease delays. It has considered different significances related to a large size of traffic practices. Additionally, this work will guide us to enhance traffic solutions within massive requests over outsized networks. Systematically, it has focused on the traffic flow, starting from the accessing steps until passing on requests to suitable spectrum carriers.
\end{abstract}

Keywords-Component; traffic predictions; software defined multiple access; dynamic spectrum sharing; $5 G$ networks

\section{INTRODUCTION}

Wireless technologies are increasingly being improved over a variety of scopes, with some of these improvements going far beyond the current needs of users. Reasons pushing this rapid growth are mostly due to either enhancing capacity or reducing latency. There is an obvious need to provide more services for different users without consuming more resources. Enormous applications need to be guaranteed in order to satisfy more users and this has simultaneously increased the cost of the providing the systems necessary for these applications. Thus, traffic issues are considered one of the biggest challenges facing the next generations. Accordingly, systems must achieve the delivery of different services by considering both the system's capacities and quality of service.

The importance of traffic predicting is to balance between a system's capacities and a user's requirements. It can manage to lessen the effects of the system's limitations. It is important to plan using a system's resources as well as to provide services for different users equally. Moreover, predicting traffic assures the correct resource allocations for enormous systems.

Different $5 \mathrm{G}$ networks have promised to provide different services in the best possible way for a variety of circumstances. Hence, it is an important to keep the delay at the minimum rate. As a result, this work has focused on enhancing the performance of $5 \mathrm{G}$ networks as well as the quality of service. It balances between both growing number of services and the large amount of users simultaneously. Primarily, choosing efficient traffic techniques improves user's experience and the quality of the provided services.

Principally, this work investigates predicting traffic by employing the recent technologies of different traffic concerns. Furthermore, it adopts fairness practices for dynamic spectrum sharing. This approach would assure the best delivery of provided services to end users by managing the available resources. It deals with each service as an individual request, and then current and previous requests are measured for forthcoming reactions. This solution will consider different requirements for providing fair spectrum sharing to enable easier service delivery. Thus, Section II presents the state of the art of data traffic management. After that Section III presents the traffic prediction model. Moving forward to operational scenarios as well as indicative results is presented in Section IV. Then, the analytical section is presented in Section V. Finally, Section VI presents the conclusion and further directions.

\section{StATE OF THE ART}

This section discusses the data traffic management in $5 \mathrm{G}$ networks. Also, it presents the concept of SoDeMa as well as operational behaviors of SoDeMa. Furthermore, it would cover the concept of spectrum sharing for different scenarios.

\section{A. Data Traffic in $5 G$ Networks}

Recently, traffic management has been raised with different fragments of large systems while traffic issues are being taken into consideration when designing large applications. Also, chosen data transmission mechanism has been gathering greater attention in recent developments, and challenging considerations have been presented by several researchers in the $5 \mathrm{G}$ era. Network traffic is expected to increase every nine months. As a reason, traffic loads will be increased over the next few years [1]. This massive growth causes raising access control issues for large applications. Several schemas have been proposed for managing traffic. For example, authors of [2] have taken on the traffic issues using different mechanism by Software-Defined Networks (SDN). They have shown the significance proposing new traffic management mechanism to enhance the quality of services. Once more, the growth of connected devices and their consequential needs is studied by authors of [3]. Therefore, an SDN controller is proposed for controlling dense networking requirements. 


\section{B. Software-Defined Multiple Access}

In the massive applications's growth, decentralized management was a precise and significant solution for network management. Recently, several types of research have offered different mechanisms, including Non-orthogonal Multiple Access NOMA instead of orthogonal access. Mainly, NOMA was proposed to increase the spectral efficiency [4]. However, for flexibility purposes, SoDeMa is recommended in terms of access management schemas within expandable networks [5]. Though both SoDeMa and NOMA achieve retrieving tasks, but SoDeMa can enable an easier configurability. Obviously, this technique can be implemented by choosing an appropriate access technique. Fig. 1 demonstrates SoDeMa with different access mechanisms. However, both availability and user's requirements will indicate the most suitable schema.

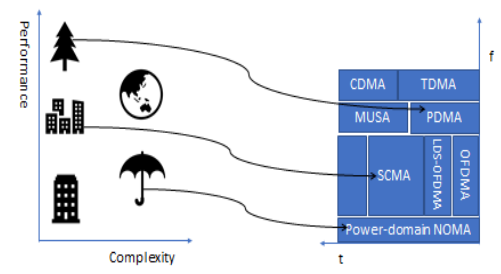

Fig. 1. SoDeMa design [5].

\section{Different Uses of SoDeMa}

SoDeMa is mainly to enhance numerous features of flexible programmability solutions. In fact, it was mainly proposed for providing higher resource allocations in large applications. It has mostly used several features from both NOMA and Software Defined Radio SDR. Thus, it efficiently allows requests to use appropriate NOMA schemas. Furthermore, SoDeMa enhances the quality of service alongside with supporting many services through $5 \mathrm{G}$ standards thus providing more options for applications to use the suitable schema.

\section{Spectrum Sharing in $5 G$}

In fact, $5 \mathrm{G}$ networks have undertaken serving enormous amounts of users in different applications types. Though, this can increase data traffic issues in large environments. Correspondingly, it requires efficient techniques of different resources to be effectively allocated. One of these resources is a spectrum that is defined as "precious limited resources required to build, maintain and expand of the Information and Communications Technology (ICT) infrastructure of any nations" [6]. Thus, efficient strategy and procedures for managing spectrum are significant points of deployment and coexistence of wireless technologies, services, and applications. Furthermore, spectrum management is a sensitive task that deals with incredible numbers of users and devices. Techniques of sharing spectrum are key facts for satisfying large applications users. Spectrum sharing has been deployed in several approaches based on the topology used and the user's preferences. Commonly, spectrum sharing is explored more fully in the following scenarios:

1) Licensed Approach: Spectrum in this scenario is allocated as primary use to wireless systems. Homogeneous horizontal sharing is the only relevant scenario of spectrum sharing. It is mainly considered as the least space of sharing among other modes since it has two methods to achieve sharing, namely limited spectrum pool as well as mutual renting. Mutual renting acts with bands of spectrum resources as subdivided blocks. Each block is licensed to a particular operator. The actual spectrum sharing occurs at this point since it operates mutually and can allow for renting limited parts of their licensed resources. Simultaneously, operators can rent resources from several other operators. However, the genuine owner of a resource has the highest level of priority among the operators accessing its resources. Limited spectrum pool allows operators to obtain a license to facilitate the band on shared basis but with limited numbers of authorized users. It could give an indication for current users of others' needs and agreements to provide more transparency.

2) Licensed Shared Access Approach: Initially, spectrum in this mode is licensed. Thus, the unused spectrum of an incumbent user can be accessed at particular times and locations. In fact, well-defined conditions determine the decision of sharing spectrum. Licenses tended to be active for long-term agreements. However, because of updates, the level of agreement varies. Thus, agreements need to be reissued. Several developments have evolved to manage the time and location preferences, and several reports have been published to enhance this mode. In fact, this approach has been leveraged from the full licensed approach but with more regulations for future systems.

3) Unlicensed Approach: In the unlicensed mode, the spectrum is wirelessly shared with other unlicensed systems. Thus, it enables more flexibility but with more required sharing regulations since this mode involves the most known scenarios of spectrums sharing cases. In this approach, both heterogeneous and homogeneous horizontal sharing modes are combined. Additionally, vertical sharing is done in the case of existing primary users. Therefore, this mode has multiple deployment scenarios and then needs more regulations to manage the perfect sharing among different systems. Different schemas have been proposed for dealing with spectrum sharing issues. Thus, several works have been using NOMA approaches in order to enable for more connectivity and massive users requirements [7]. Furthermore, others are combining both NOMA with cognitive radio technologies to leverage from both technologies in heterogeneous networks [8], [9]. However, further works have been proposing other techniques to enable the best of shared spectrum among $5 \mathrm{G}$ networks based on reconfigurable SDN technologies in massive devices [10]-[12]. Also, other researchers have focused on D2D based spectrum sharing for vehicular purposes to enable massive connections such as [13]. The spectrum is 
envisioned that $5 \mathrm{G}$ will be 10 times improved to satisfy the 1000 times of throughput improvements [14].

\section{TRAFFiC PREDICTING MODEL}

This model focuses on predicting traffic of different requests for enabling an easier spectrum sharing among large systems. Thus, we will cover the entire process of the traffic predicting model from the beginning to assigning to the suitable spectrum sharing provider. Predicting traffic in $5 \mathrm{G}$ networks involves examining different reasons for solving this issue. Therefore, our solution enhances traffic predicting in different ranges. This means we have considered suitable accessing schemas, for serving user's needs at the best of resource saving. Also, it has considered the Quality of service in terms of spectrum sharing. In addition, it has focused on each slot of time with it served for estimation purposes.

This work applies two factors, starting by calculating the exact duration for each request in order to use the suitable spectrum carrier. This step is significant to allocating network resources. Consequently, requests are considered based on different ranges of priority to calculate the needed time. Requests mainly fall under the offered bandwidth within this system. It is a key point that a serving request goes to the nearest slot of time. Thus, it also accounts for the number of requests for future traffic issues. At this time, requests are classified by the traffic controller, based on the needed time and their types. Furthermore, this controller assigns each request to an appropriate accessing mechanism after calculating the time needed. However, spectrum carrier is individually considered based on its capacity. Consequently, it is necessary to identify the capacities of carriers to deal with future requests. Afterward, the assigning step assures accounting for both times needed for each request. Then, it calculates the traffic at the particular slot of time for calculating future traffic. Accordingly, previous steps are considered as the first scenario of this model.

The traffic controller assigns different requests to the needed service. Then, it leverages from current services for future estimations. Users are served based on their needs as well as the available resources. Before assigning to the spectrum carrier, decisions are already made by the controller for enabling an easier resource allocation. The history of previous requests is saved to estimate future traffic approximations. The future estimations are based on both location and times of requests. Thus, frequent requests are classified into their locations and the slot of time to be served with suitable spectrum carrier in future experiences.



Fig. 2. An entire system model.
Our model is divided into two main scenarios. Scenario one internally measures traffic to provide requests with the time approximations as shown in Fig. 2. However, scenario two has calculated different factors of current requests for future traffic purposes. Therefore, it will consider the most frequent carrier for future purposes. It will not repeat the calculations of scenario one for the future calculated request. Thus, recording the most frequent decisions will be saved for specific requests. In fact, this step would help for more resource savings and better allocations. Generally, this includes implementing two steps in order to deal with future requests. Since scenario one will deal with the new requests, however scenario two analyzes the previous requests as well as their requirements based on the accessible resources.

In fact, replication process of scenario one is avoided by calculating frequently received requests. Moreover, it will assist in reducing the consumption of systems resources. Then, it would decrease the needed time when serving requests. This would enable for an easier decision making especially for a massive number of users to the suitable spectrum provider. Thus, a particular spectrum carrier will be assigned by the time of the systems. Therefore, it benefits both asynchronous requests and requests with real-time requirements. Different schemas will be classifies to serve frequent requests. Also, timestamps will help in determining the frequent request and the suitable used schemas.

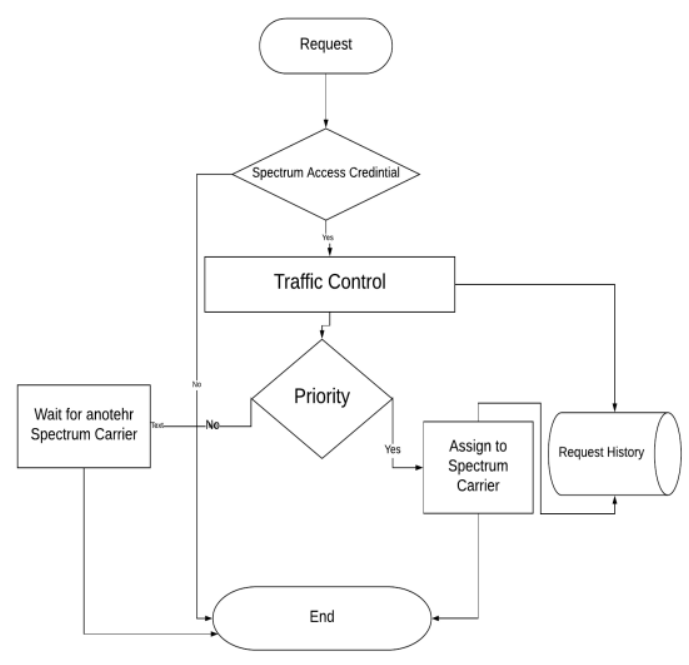

Fig. 3. Simple request process of dynamic spectrum sharing.

As seen in Fig. 3, a simple process is used for incoming requests. In the beginning, it would be treated at the level of credentials. If this request is authorized, it would be processed to the next step. Then, after the request has been processed, the traffic control will be in charge of dealing with this request based on its previous experiences. During the first time, this request will be recorded for future purposes. However, if the coming request is regular, the control will check with its stored previous experiences. This will help determine the initial priority needs. Next, this request will be processed further in the priority stage. In the priority step, the request will be treated based on its previous and current needs. This step accounts for the accurate traffic levels for better decision 
making. Then, again, it will assign the request to most suitable spectrum sharing. For enabling dynamic sharing, each request is examined twice while processing for enabling more dynamicity of spectrum sharing. In fact, requests are served with an easier technique for analyzing previous experiences. The difference can be recognized through the benefit of using previous requests for future optimizations. Thus, it is focused on choosing the suitable spectrum sharing to manage the overall network traffic.

Moving to the main focus, estimating future traffic to enable better decision support, we have to consider the situations of past requests. Thus, the time taken for the previous request will be an important factor for our upcoming requests. This has benefits in the managing of resources and predicting the needs of current requests. However, the predicting phase depends on available bandwidth as well as the priority of the request. These predictions will be based on both the slot of time given as well as the network experience of previous requests. Henceforth, it helps in future traffic estimations to assign the incoming requests to particular carriers.

\section{OPERATIONAL SCENARIOS AND INDICATIVE RESUlTS}

This section describes the showcases of Estimating Data Traffic, followed by indicative results for managing the traffic among heterogeneous networks by using SoDeMa technology.

\section{A. Scenario 1: SoDeMa}

As presented in Section II, SoDeMa can play an important role in managing traffic within enormous networks, by enabling flexible configuration when choosing the suitable access schema. However, in this proposal, it is very important to manage the traffic to assign the incoming request to a suitable spectrum carrier. Thus, we will be discovering several schemas where SoDeMa can be adopted for enhancing the overall throughput as well as managing the traffic issues. We are assuming that several users with different requests wish to access an application with different privileges. Our scenario will focus on providing them with the best service while considering their bandwidth capacities. To be clear, overall latency requirements must be considered for service estimations. However, it is also obvious that showing current needs is significant for priority classifications. Users are informed by messages for spectrum capacities. Once the channel is free for incoming requests, users will be informed for assigning them to a suitable spectrum carrier.

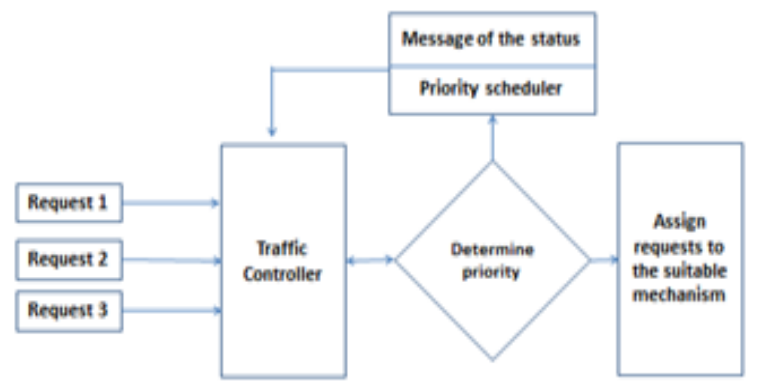

Fig. 4. SoDeMa architecture in $5 \mathrm{G}$ environments.
As seen in Fig. 4, incoming requests will be assigned to the traffic controller. This step can measure internal traffic to provide users with the service approximations. Also, this controller determines the priority of needed services in a dynamic method. The controller notifies users of the traffic status. However, to estimate the time of achieving different services, users will be notified by a message including both status and times needed. Therefore, a notifying message will be suitable for time management as well as for priority purposes.

Accordingly, at this step, computing the time needed for each request would be critical factor. All requests are individually considered based on the different priority levels. Thus, the available spectrum carriers results on serving different requests. Furthermore, it is an important to serve requests within the nearest slot of time. The controller will record the number of requests for future traffic purposes. This controller assigns the request to the suitable spectrum carrier after calculating the needed time. Accordingly, the capacities of available spectrums needs to determined to deal perfectly with current requests. In fact, this step assures calculating both the traffic within the slot of time and the time needed for each request.

\section{B. Scenario 2: Predicting Data Traffic}

At scenario 1, it is an important to calculate the time needed for serving each request, depending on both priority and the available spectrum. Hence, this scenario focuses on additional aspect of the traffic process. Thus, it calculates the most frequent spectrum carrier for flexible future assigning steps. Thus, it will overcome the calculations of scenario 1 for the future request. Therefore, recording repeated notifying message will be saved for particular requests. This scenario was achieved by implemented two steps as presented below:

1) Frequent requests: At this step, the time slot of the system's availability will be assigned to particular multiple accesing schemas. Thus, it helps supporting asynchronous requests or requests with real time needs. Thus, frequent requests will be kept as an array in the system's reccords. Requests will be identified by the time stamp to extract frequent requests. As a result, this step analyzes the current situation of the request and provides the history of decisions made in order to support future choices. Also, it will focus on the time of the request to for priorities practices.

2) Calculating data volume: It has considered the situations of past requests, in order to estimate future traffic. Thus, it enables for better decision making. Therefore, the previously time taken acts as a significant factor in all the future requests. Urgent requests will be directly assigned to a spectrum carrier. Thus, estimation steps firstly depend on both slot of time, then on the experience of spectrum sharing. Thus, the future traffic decisions are estimated by returning past made decisions of similar request.

\section{Scenario 3: Dynamic Spectrum Sharing}

Previous scenarios have mainly participated in achieving this scenario for dynamic sharing of spectrum. This supports an integrated solution in our proposal. For dynamicity support, SoDeMa enhances decision making for choosing the best 
available carrier. This decision is made according to different factors. These factors include time taken for the previous request, quality of experiences and so on. Individually, each request is considered in order to provide the best service as well as taking into consideration the quality of service. Furthermore, in scenario 2, predicting data traffic enhances the current status for the systems to estimate future traffic based on current traffic management as well as the current system's capacity. Thus, this scenario allows dynamic sharing of the spectrum based on past request experiences. However, it leverages the current requests for two reasons. The first is for predicting the traffic in the future. The second is for recording the current traffic of requests based on the time of the request as well the time taken for achieving this request.

As seen in Fig. 5, the scenario of dynamic spectrum needs to be shared perfectly. Thus, requests need an optimized assigning at the end of the process. The control unit classifies carriers into four main types. Green color means the carrier is ready for receiving the new request based on its capacity. A black path means the carrier is currently available, but another request just has been assigned to share this spectrum. Furthermore, a red color means this carrier is not available at this time and serving another request. The time taken for serving this request is very important for the next request to perfectly manage the time. The blue color means this carrier is prepared for another request and this color also provides the capacity limitations for dealing with new requests. Dynamically, colors change based on the current situations of the spectrum carrier. Thus, each status is understood by the traffic controller and the approximations of time. Colors are changed based on the notifying messages sent continuously to the controller. This approach will assure that all requests are treated equally, and at the same time, the status of the spectrum and providers are accurate.

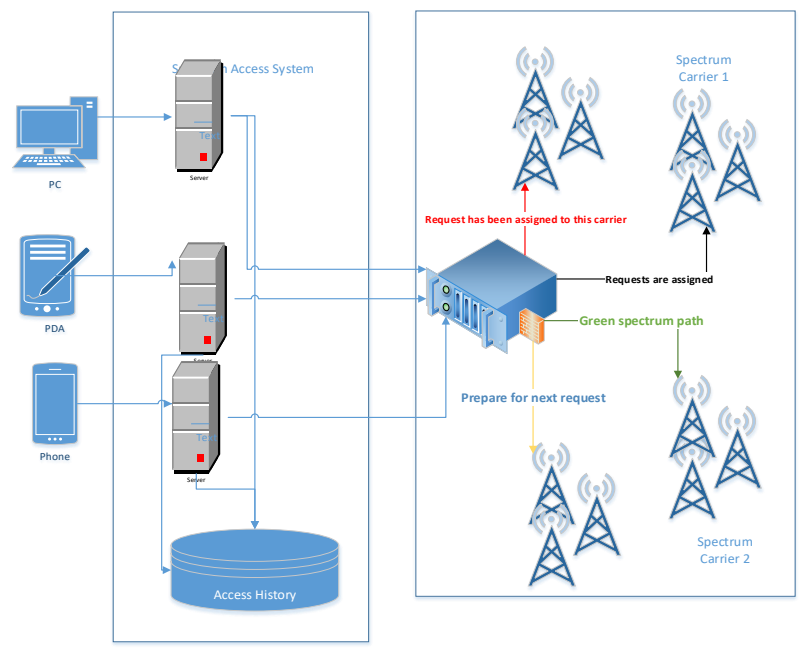

Fig. 5. Dynamic spectrum sharing scenarios.

\section{ANALYSIS}

Traffic conditions are a significant factor in this work. This section analyzes the proposed model in order of predicting traffic. In fatc, this work is focused to be implemented on the future $5 \mathrm{G}$ networks. Therefore, we focused on several factors including spectrum sharing, time, packet loss, and frequent request reactions. Significantly, it is important to study the existing traffic solutions for investigating their usages within SDN and SoDeMa.

\section{A. Time Management}

This work invitistaiges traffic predicting by recent technologies for heterogeneous networks. Thus, time management is a crucial aspect and must be addressed for traffic enhancements. As a consequence, time management for enormous numbers of requests is an identical issue. Hence, it has focused on keeping the delay of requests at the minimum rates. Also, the time taken is considered in all steps of this work. Correspondingly, this solution ensures reducing time consumptions for future networks by predicting the traffic approximations, which will help decision making of frequent requests. Again, it has prevented the repetition of scenario 1 steps for frequent incoming requests. Also, classifying the user's services is implemented to assign them to the suitable spectrum provider.

\section{B. Spectrum Sharing Schema}

Another important issue has been dicovered in our model. This was regarding spectrum sharing which was solved by distributing the service colors. It was classified by based on the availability and frequently booked channels, as shown in Fig. 5. Thus, it is classified into four main colors, for representing different scenarios. This has helped classify the different users as well as to estimate the time of assigning the desired service to the suitable carrier. On the other hand, priority considerations were considered by implementing the quality of service of different users. In fact, several factors are affected in this issue including the large and various services. Also, the changeability of available spectrums is another factor at this step. For future considerations, each request is maintained by the needed time of delivering services.

\section{Packet Loss}

Huge data transmission could result on packet loss, which can be considered as a bottleneck in 5G systems. This is caused by the increasing number of users as well as the hug data traffic. This system has overcome this issue by estimating time for frequent users. Thus, the frequent user is given an estimated time before resending packets. Mainly, this solution is implemented by giving an approximate time before sending any packet. The time given is calculated by consdiering several factors as well as the available spectrum. Thus, requests will be given a range of time until it is serve, otherwise it will resend the lost packets.

\section{Frequent Request Considerations}

Mainly, the proposed model focuses on saving both time and network resources. Thus, requests at the first time will be served, but will not be considered as frequent ones. They, will be served and then enter the chain of previous request for better decision making in fture. After serving first time request, the next times will be easier for the systems to enable them with frequent user'sconsiderations. At the next steps, it will enable for more flexibility since scenario one procedures is avoided. However, they will be saved for the network records for future purposes. Though, it will not solve all the issues of coming 
requests but at least will consider frequent users request and then enable for a faster resources allocating.

\section{CONCLUSION AND FURTHER DIRECTIONS}

In conclusion, this model has investigated traffic predicting in $5 \mathrm{G}$ networks. Thus, It has proposed predicting data traffic by using a new technology, called SoDeMa. This dynamically enhances spectrum sharing process. Moreover, it has concluded that managing traffic, starting from the access schema would improve the throughput of large applications within 5G networks. It can be considered as an efficient approach to track the current traffic issues. The main difference in this model is to overcome the replications of dealing with different requests in future experiences. The outlined advantages of this paper are to solve traffic management through a new sharing mechanism. Thus, applying this technique would insure flexible services in heterogeneous networks. Also, it can flexibly achieve requests of large numbers of users.

Future directions are mainly based on the new requirements of the next generation. Coming generations will be mainly focusing on enhancing the service provided. Other attempts should investigate the load of traffic prediction on the overall throughput. Finally, the changeability status must be accurate for enabling more efficiency when applying dynamic sharing among spectrum carriers.

\section{REFERENCES}

[1] B. Bangerter, S. Talwar, R. Arefi, K. Stewart, "Networks and devices for the 5G era", IEEE Comm. Mag., vol. 52, no. 2, pp. 90-96, Feb. 2014.

[2] K. Kosek-Szott et al., "Coexistence Issues in Future WiFi Networks," in IEEE Network, vol. 31, no. 4, pp. 86-95, July-August 2017.

[3] A. Muthanna, R. Gimadinov, R. Kirichek, A. Koucheryavy and M. S. A. Muthanna, "Software development for the centralized management of IoT-devices in the "smart home" systems," 2017 IEEE Conference of Russian Young Researchers in Electrical and Electronic Engineering (EIConRus), St. Petersburg, 2017, pp. 190-194.
[4] J. Choi, "NOMA: Principles and recent results," 2017 International Symposium on Wireless Communication Systems (ISWCS), Bologna, Italy, 2017, pp. 349-354.

[5] Dai. L., Wang, B. Yuan, Y. Han, S., I, C. and Wang, Z., ” Nonorthogonal multiple access for 5G: solutions, challenges, opportunities, and future research trends". IEEE Communications Magazine, 2015, 53(9), pp.74-81.M. Young, The Technical Writer's Handbook. Mill Valley, CA: University Science, 1989.

[6] R. B. F. Da Silva and C. T. R. Da Silva, "Spectrum regulation in Brazil," IEEE Wireless Communications, vol. 23, no. 3, pp. 2-3, 2016.

[7] L. Lv, J. Chen, Q. Ni, Z. Ding and H. Jiang, "Cognitive Non-Orthogonal Multiple Access with Cooperative Relaying: A New Wireless Frontier for 5G Spectrum Sharing," in IEEE Communications Magazine, vol. 56, no. 4, pp. 188-195, APR, 2018.

[8] Y. Liu et al., "Nonorthogonal Multiple Access In Large-Scale Underlay Cognitive Radio Networks", IEEE Trans. Vehic. Technol., vol. 65, no. 12, pp. 10152-57, Dec. 2016.

[9] L. Lv et al., "Design of Cooperative Non-Orthogonal Multicast Cognitive Multiple Access for 5G Systems: User Scheduling and Performance Analysis", IEEE Trans. Commun., vol. 65, no. 6, pp. 264156, June 2017.

[10] F. Mekuria and L. Mfupe, "Spectrum sharing \& affordable broadband in 5G," 2017 Global Wireless Summit (GWS), Cape Town, 2017, pp. 114-118.

[11] X. Duan, X. Wang, L. Zhang, W. Li and Y. Wu, "Software defined orchestrated spectrum sharing enabled by 3D interference map," 2017 IEEE International Symposium on Broadband Multimedia Systems and Broadcasting (BMSB), Cagliari, 2017, pp. 1-6.

[12] X. Duan, X. Wang, "Authentication handover and privacy protection in $5 \mathrm{~g}$ hetnets using software-defined networking", IEEE Communications Magazine, vol. 53, no. 4, pp. 28-35, April 2015.

[13] J. Liu et al., "Device-to-Device Communication in LTE-Advanced Networks: A Survey", IEEE Commun. Surveys \& Tutorials, vol. 17, no. 4, pp. 1923-40, 2015.

[14] L. Zhang, M. Xiao, G. Wu, M. Alam, Y. C. Liang and S. Li, "A Survey of Advanced Techniques for Spectrum Sharing in 5G Networks," in IEEE Wireless Communications, vol. 24, no. 5, pp. 44-51, October 2017.

[15] A. Alshaflut and V. Thayananthan, "Estimating data traffic through software-defined multiple access for IoT applications over 5G networks," 2018 15th Learning and Technology Conference (L\&T), Jeddah, 2018, pp. 59-66. 